\title{
Extensions of supersymmetric spin systems
}

\author{
H Nicolai \\ Institut für Theoretische Physik, Universität Karlsruhe, Postfach 6380, Kaiserstrasse 12, \\ 75 Karisruhe 1, FDR
}

Received 15 June 1977

\begin{abstract}
A discussion of supersymmetric spin systems is presented extending the results obtained in a recent paper. A two-dimensional model is given and gauge invariance is defined; the latter is shown to necessitate the introduction of further spin operators.
\end{abstract}

\section{Introduction}

In a recent paper (Nicolai 1976, to be referred to as I) it was shown that supersymmetry (graded Lie algebras) may be applied to non-relativistic systems containing bosonic and fermionic variables such as (an)harmonic oscillators and spins. In this article we want to extend some of the results obtained in I. As in I the basic algebra is

$$
\begin{aligned}
& \{Q, Q\}=\left\{Q^{+}, Q^{+}\right\}=0 \quad\left\{Q, Q^{+}\right\}=H \\
& {[Q, H]=\left[Q^{+}, H\right]=0}
\end{aligned}
$$

where $Q$ and $Q^{\dagger}$ generate supersymmetry transformations and $H$ is the Hamiltonian of the system. As was shown in I, non-trivial realisations of (1.1) may be constructed in a systematic fashion by introducing the concept of a 'superfield' $\phi(t, \theta, \bar{\theta}) \dagger$ which is a function of $t \in \mathbb{R}$ and two anticommuting elements $\theta$ and $\bar{\theta}$ of a Grassmann algebra. The Taylor expansion of $\phi$ in $\theta$ and $\bar{\theta}$ terminates after a finite number of terms because $\theta^{2}=\bar{\theta}^{2}=0$; the physical quantities of a model are to be associated with the coefficients of $\theta$ and $\vec{\theta}$ in this expansion. A superfield in itself may be either an even or an odd element of the Grassmann algebra and is then said to 'commute' or to 'anticommute', respectively. In I it was shown that commuting superfields may be used to construct systems containing spins and harmonic oscillators whereas anticommuting superfields can be used to construct pure spin systems with non-trivial interactions. Only the latter will be considered in this article.

As was argued in I, the use of anticommuting operators appears to be indispensable for supersymmetry to make sense; this, however, presents a difficulty when one considers dimensions $d \geqslant 2$. For $d=1$, the connection between Fermi and Pauli operators was rather free from ambiguities as a 'natural' ordering of the spins was given. For $d \geqslant 2$, there is no uniquely 'natural' way to relate Fermi and Pauli operators although the transcription may still be performed, the number of spins being

$\uparrow$ The general concept of a superfield is due to Salam and Strathdee (1974). 
denumerable for $d<\infty$ (Lieb et al 1961). For instance, on a two-dimensional lattice we have

$$
\bar{\psi}_{j} \psi_{j+\hat{n}}=\exp \left(\mathrm{i} \pi \sum_{l \in \boldsymbol{Q}_{, i, i}+\hat{A}} s_{l}\right) \sigma_{j}^{+} \sigma_{j+\hat{n}}^{-}
$$

where $\mathscr{C}_{i, j+\hat{n}}$ is the curve connecting $j$ and $j+\hat{n}$ when enumerating spins and $s_{l}=$ $\frac{1}{2}\left(\sigma_{l}^{3}+1\right)$ is the occupation number of spin $l$ (figure 1 ). In general, we may not expect the exponential factors to cancel; only for particular interactions such as $\bar{\psi}_{i} \psi_{j} \bar{\psi}_{j+\hat{n}} \psi_{j+\hat{n}} \sim \sigma_{i}^{3} \sigma_{j+\hat{n}}^{3}$ these undesired factors cancel out (another example is shown in figure 2). Thus, it appears that none of the models of this paper for $d \geqslant 2$ is in any direct and obvious fashion related to usual $d$-dimensional Heisenberg models although one should not exclude the possibility of their thermodynamic properties resembling each other (this is the main motivation for writing this article). Consequently, we propose to study supersymmetric spin models in their own right; supersymmetry which seemingly cannot be formulated for usual spin models with $d \geqslant 2$ may then yield insights which could not be obtained otherwise.
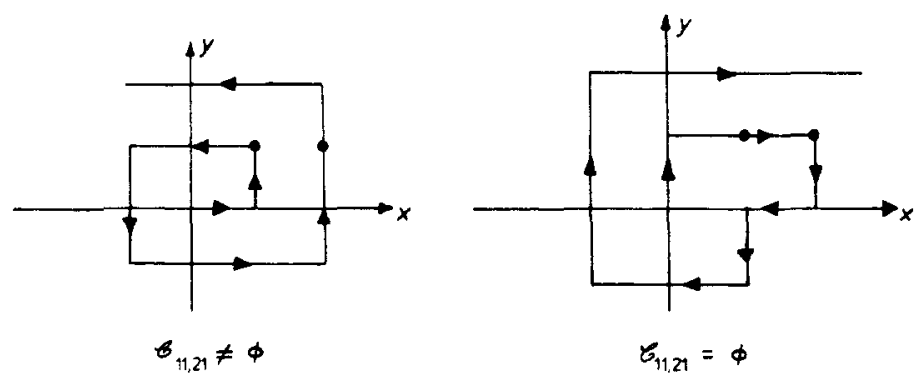

Figure 1. Two ways of enumerating spins on a two-dimensional lattice.

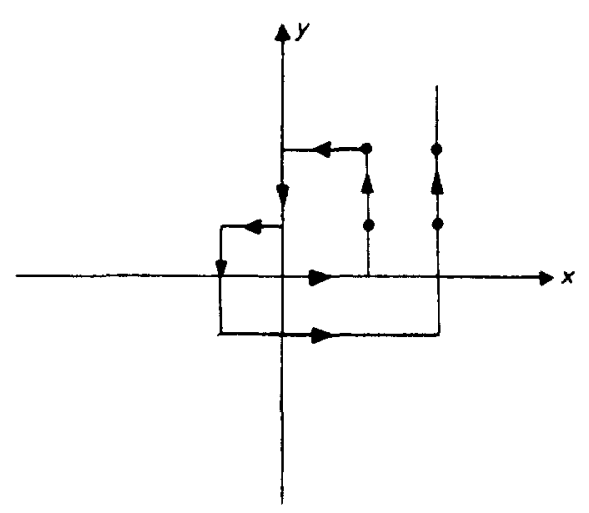

Figure 2. An example of a four-spin interaction where unwanted factors cancel.

In $\S 2$, a generalisation of the spin model given in $I$ for $d=2$ is presented and a ' $X Y$-type' model $\dagger$ is shown to be obtainable by going to a singular limit. In $\S 3$, gauge symmetry is introduced-here, solely for simplicity, we restrict our attention to the case $d=1$ mainly and indicate how to generalise to $d \geqslant 2$. All calculations will be

$\dagger$ By abuse of language we call $\bar{\psi}_{i} \psi_{i+\hat{n}}+\mathrm{HC}$ an ' $X Y$-type' interaction; no confusion should arise when we make use, freely but not always correctly, of conventional terminology. 
made using Lagrangians and regarding Grassmann numbers as the 'classical' analogues of spin operators. After changing to the Hamiltonian, these 'classical' spins are quantised and can only then be interpreted physically. In appendix 1 the possible consequences of supersymmetry for correlation and 'pseudo-correlation' functions are discussed more thoroughly than in I; in appendix 2 , we show how to reconstruct the generators of supersymmetry from a given Lagrangian.

For the convenience of the reader we explain here the notation that will be needed in the following. Superfields will be denoted by $X_{j}, \Lambda_{k}, V_{l}, \ldots$, where $j, k, l, \ldots$ are lattice indices. The expansion coefficients of superfields in $\theta$ and $\bar{\theta}$ will be denoted by $a_{j}, c_{k}, v_{l}, \ldots$ if they commute and by $\chi_{i}, \lambda_{k}, \psi_{l}, \ldots$ if they anticommute.

\section{Two-dimensional model}

We introduce a set of anticommuting constrained superfields $\left\{X_{i}\right\}$ (for details $\mathrm{cf}$ I)

$$
\begin{array}{ll}
\overline{\mathrm{D}} X_{2 j}=0: & X_{2 j}=\chi_{2 j}+\theta a_{2 j}+\frac{1}{2} \mathrm{i} \theta \bar{\theta} \dot{\chi}_{2 j} \\
\mathrm{D} X_{2 j}^{*}=0: & X_{2 j}^{*}=\bar{\chi}_{2 j}+\bar{\theta} a_{2 j}^{*}-\frac{1}{2} \mathrm{i} \theta \bar{\theta} \dot{\bar{\chi}}_{2 j} \\
\overline{\mathrm{D}} X_{2 j+\hat{n}}=0: & X_{2 j+\hat{n}}=\bar{\chi}_{2 j+\hat{n}}+\theta a_{2 j+\hat{n}}^{*}+\frac{1}{2} \mathrm{i} \theta \bar{\theta} \dot{\bar{\chi}}_{2 j+\hat{n}} \\
\mathrm{D} X_{2 j+\hat{n}}^{*}=0: & X_{2 j+\hat{n}}^{*}=\chi_{2 j+\hat{n}}+\bar{\theta} a_{2 j+\hat{n}}-\frac{1}{2} \mathrm{i} \theta \bar{\theta} \dot{\chi}_{2 j+\hat{n}}
\end{array}
$$

where now, in contrast to I, we take the lattice to be two-dimensional $\ddagger$ (figure 3):

$$
\begin{array}{ll}
j=\left(j_{1}, j_{2}\right) ; & 1 \leqslant j_{i} \leqslant N \\
\hat{n}=\hat{1}, \hat{2} ; & \hat{1}=(1,0), \hat{2}=(0,1) .
\end{array}
$$

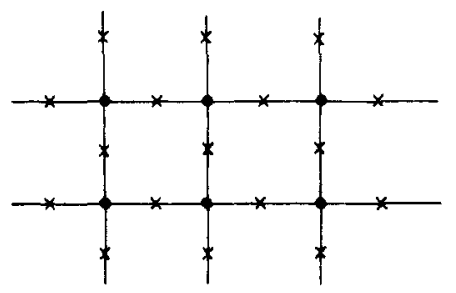

Figure 3. Lattice $G$ and location of spins; the full circles are for spins $2 j$ and the crosses for $2 j+\hat{n}$.

As was explained in I, invariants (under supersymmetry transformations) may be obtained by mutliplying superfields and extracting those terms that transform as total derivatives with respect to $t \in \mathbb{R}$.

The basic invariant containing $X_{i}$ and $X_{j}^{*}$ is $(j \in G)$ :

$$
\frac{\partial^{2}}{\partial \bar{\theta} \partial \theta} X_{j} X_{j}^{*} \cong \mathrm{i} \dot{\chi}_{i} \bar{X}_{i}+a_{i}^{*} a_{i} \quad \text { (or } \mathrm{i} \dot{\bar{\chi}} \chi_{i}+a_{i}^{*} a_{j} \text { ) }
$$

$\dagger$ The covariant derivatives $\mathrm{D}$ and $\overrightarrow{\mathrm{D}}$ are given by (I):

$$
\mathrm{D}=\frac{\partial}{\partial \theta}+\frac{1}{2} ; \bar{\theta} \frac{\mathrm{d}}{\mathrm{d} t} \quad \text { and } \quad \overline{\mathrm{D}}=-\frac{\partial}{\partial \bar{\theta}}-\frac{1}{2} \mathrm{i} \theta \frac{\mathrm{d}}{\mathrm{d} t} .
$$

$\ddagger$ As in I we assume periodic boundary conditions. 
('kinetic term'). The most general interaction Lagrangian is

$\mathscr{L}_{\text {int }}=\left(\sum_{j \in G} g_{j} X_{i}+\sum_{i, j, k \in G} g_{i j k} X_{i} X_{j} X_{k}+\sum_{i, j, k, l, m \in G} g_{i j k l m} X_{i} X_{j} X_{k} X_{l} X_{m}+\ldots\right)_{a-\text { component }}+\mathrm{HC}$

where $g_{i j k} g_{i j k l m}, \ldots$ are totally antisymmetric and HC stands for Hermitian conjugate. Note that terms containing an even number of $X$ 's lead to anticommuting invariants and therefore are not included in (2.4).

As our model we take $(f, g \in \mathbb{R})$

$\mathscr{L}_{\text {int }}=\left(f \sum_{i \in G} X_{i}+g \sum_{j=\left(j_{1}, j_{2}\right)}\left(X_{2 j} X_{2 j+\hat{1}} X_{2 j+2 . \hat{1}}+X_{2 j} X_{2 i+\hat{2}} X_{2 j+2 . \hat{2})}\right)_{a-\text { component }}+\right.$ HC.

Written out, this is

$$
\begin{aligned}
& \mathscr{L}_{\text {int }}=f \sum_{j=\left(j_{1}, j_{2}\right)}\left(a_{2 j}+a_{2 j}^{*}+a_{2 j+\hat{1}}+a_{2 j+\hat{1}}^{*}+a_{2 j+2}+a_{2 j+\hat{2}}^{*}\right)
\end{aligned}
$$

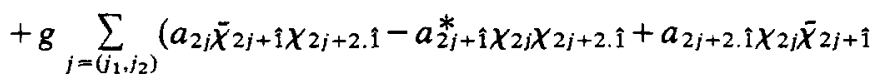

$$
\begin{aligned}
& +a_{2 j}^{*} \bar{X}_{2 j+2 . \hat{1}} \chi_{2 j+\hat{1}}-a_{2 j+\hat{1}} \bar{X}_{2 j+2 . \hat{1}} \bar{X}_{2 j}+a_{2 j+2 . \hat{1}}^{*} \chi_{2 j+\hat{1}} \bar{\chi}_{2 j} \\
& +a_{2 i} \bar{X}_{2 j+2} \chi_{2 j+2.2}-a_{2 j+2}^{*} X_{2 j} \chi_{2 j+2.2}+a_{2 j+2.2} X_{2 j} \bar{X}_{2 j+2}
\end{aligned}
$$

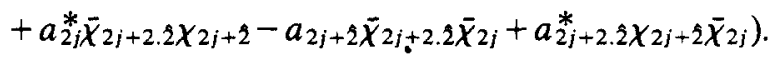

From $\mathscr{L}=\mathscr{L}_{\text {kin }}+\mathscr{L}_{\text {int }}$ we obtain the equations of motion:

$$
\begin{aligned}
& -a_{2 i}^{*}=f+g \bar{\chi}_{2 j+1} \chi_{2 j+2 . \hat{1}}+g \chi_{2 j-2.1} \bar{\chi}_{2 j-\hat{1}}+g \bar{\chi}_{2 j+2} \chi_{2 j+2 . \hat{2}}+g \chi_{2 j-2.2} \bar{X}_{2 j-2} \\
& -a_{2 j+\hat{1}}^{*}=f-g \bar{X}_{2 i+2 . \hat{1}} \bar{X}_{2 i} \\
& -a_{2 j+\hat{2}}^{*}=f-g \bar{X}_{2 j+2.2 .} \bar{X}_{2 j}
\end{aligned}
$$

and similarly for Hermitian conjugates. Substituting (2.7) into (2.6) and changing to the Hamiltonian, the 'kinetic term' drops out and we are left with

$$
H=\sum_{j \in G} a_{j}^{*}(\chi, \bar{\chi}) a_{j}(\chi, \bar{\chi}) .
$$

This expression will not be written out explicitly but rather we shall consider a special case. Setting

$$
f:=\gamma g^{-1}
$$

subtracting $3 N^{2}(\gamma / g)^{2}$ (a constant) from (2.8) we can take the limit $g \rightarrow 0$ in which, clearly, supersymmetry transformations become singular. The model with results is

$$
\begin{aligned}
& \lim _{g \rightarrow 0}\left[H-3 N^{2}(\gamma / g)^{2}\right] \\
& =\gamma \sum_{\left.j=\bar{\sigma}_{1}, j_{2}\right)}\left(\bar{\chi}_{2 j-\hat{1}} \chi_{2 j}+\bar{\chi}_{2 j} \chi_{2 j-\hat{1}}+\chi_{2 i} \bar{X}_{2 j+\hat{1}}+\chi_{2 j+1} \bar{\chi}_{2 j}+\bar{\chi}_{2 j-2} \chi_{2 j}+\bar{\chi}_{2 j} \chi_{2 j-\hat{2}}\right. \\
& \left.+\chi_{2 j} \bar{\chi}_{2 j+\hat{2}}+\chi_{2 j+\hat{2}} \bar{\chi}_{2 j}-\bar{\chi}_{2 j+2 . \hat{1}} \bar{\chi}_{2 j}-\chi_{2 i} \chi_{2 j+2 . \hat{1}}-\bar{\chi}_{2 j+2 . \hat{\chi}} \bar{\chi}_{2 j}-\chi_{2 j} \chi_{2 j+2 . \hat{2}}\right)
\end{aligned}
$$


and is evidently ' $X Y$-type'. In one dimension, a similar limit leads to a $X Y$-chain of alternating ferromagnetic and antiferromagnetic type.

\section{Gauge invariance}

In this section we shall be concerned primarily with $d=1$ and therefore the indices $j$ are one-dimensional and run from 1 to $2 N$. The relativistic case is discussed in Ferrara and Zumino (1974) and we shall proceed analogously. As our starting point we choose the model (5.6) of I:

$\mathscr{L}=\mathscr{L}_{\text {kin }}+\mathscr{L}_{\text {int }}$

$$
=\text { invariant part of }\left(\sum_{i=1}^{2 N} X_{j} X_{j}^{*}+g \sum_{i=1}^{N}\left(X_{2 j-1} X_{2 j} X_{2 j+1}+\mathrm{HC}\right)\right)
$$

where, as in $\S 2,\left\{X_{i}\right\}$ are constrained anticommuting superfields (except for the indices, the notation is the same as in (2.1)). The equations of motion are

$$
\begin{aligned}
& -a_{2 j-1}^{*}=g \chi_{2 i+1} \bar{\chi}_{2 j}+g \bar{\chi}_{2 j-2} \chi_{2 j-3} \\
& -a_{2 j}^{*}=-g \bar{\chi}_{2 j-1} \bar{\chi}_{2 j+1} .
\end{aligned}
$$

Now, we introduce $N$ constrained commuting superfields $\Lambda_{2 j+1}$ :

$$
\begin{array}{ll}
\overline{\mathrm{D}} \Lambda_{2 j+1}=0: & \Lambda_{2 j+1}(t, \theta, \bar{\theta})=c_{2 j+1}(t)+\theta \lambda_{2 j+1}(t)+\frac{1}{2} \mathrm{i} \theta \bar{\theta} \dot{c}_{2 j+1}(t) \\
\mathrm{D} \Lambda_{2 j+1}^{*}=0: & \Lambda_{2 j+1}^{*}(t, \theta, \bar{\theta})=c_{2 j+1}^{*}(t)+\bar{\lambda}_{2 j+1}(t) \bar{\theta}-\frac{1}{2} \mathrm{i} \theta \bar{\theta} \bar{c}_{2 j+1}^{*}(t)
\end{array}
$$

which will parametrise gauge transformations $\ddagger$. These are defined by

$$
\begin{aligned}
& X_{2 j+1}(t, \theta, \bar{\theta}) \rightarrow \exp \left(\mathrm{i} \Lambda_{2 j+1}(t, \theta, \bar{\theta})\right) X_{2 j+1}(t, \theta, \bar{\theta}) \\
& X_{2 j}(t, \theta, \bar{\theta}) \rightarrow \exp \left[-\mathrm{i}\left(\Lambda_{2 j-1}(t, \theta, \bar{\theta})+\Lambda_{2 j+1}(t, \theta, \bar{\theta})\right)\right] X_{2 j}(t, \theta, \bar{\theta})
\end{aligned}
$$

and, obviously, (3.1) is invariant with respect to (3.4). Similar definitions hold for $X_{2 j}^{*}$, $X_{2 j+1}^{*}$. Observe that $\mathrm{e}^{\mathrm{i} \Lambda} X$ anticommutes and obeys $\mathrm{D}\left(\mathrm{e}^{\mathrm{i} \Lambda} X\right)=0$, as is required for consistency. To make the kinetic term $\Sigma_{j=1}^{N} X_{i} X_{j}^{*}$ invariant, we introduce gauge superfields $V_{2 i+1}$ and $V_{2 j+1}^{*}$ :

$$
\begin{aligned}
& V_{2 j+1}(t, \theta, \bar{\theta})=v_{2 j+1}(t)+\theta \varphi_{2 j+1}(t)+\bar{\psi}_{2 j+1}(t) \bar{\theta}+\theta \bar{\theta} w_{2 j+1}(t) \\
& V_{2 j+1}^{*}(t, \theta, \bar{\theta})=v_{2 j+1}^{*}(t)+\theta \psi_{2 j+1}(t)+\bar{\varphi}_{2 j+1}(t) \bar{\theta}+\theta \bar{\theta} w_{2 j+1}^{*}(t)
\end{aligned}
$$

which transform as follows:

$$
\begin{aligned}
& V_{2 j+1}(t, \theta, \bar{\theta}) \rightarrow V_{2 j+1}(t, \theta, \bar{\theta})-\mathrm{i} \Lambda_{2 j+1}(t, \theta, \bar{\theta}) \\
& V_{2 j+1}^{*}(t, \theta, \bar{\theta}) \rightarrow V_{2 j+1}^{*}(t, \theta, \bar{\theta})+\mathrm{i} \Lambda_{2 j+1}^{*}(t, \theta, \bar{\theta}) .
\end{aligned}
$$

Upon expanding $V$ in powers of $\theta$ and $\bar{\theta}$ and comparing coefficients we find that the $\uparrow$ In equation (5.7) of I the signs in front of $a_{2 j} \bar{\psi}_{2,-1} \bar{\psi}_{2,+1}$ and its Hermitian conjugate should read - instead of + . Equation (5.8) should be changed accordingly.

$\ddagger$ For abuse of language see remark in footnote to page 2144 . 
components of $V(t, \theta, \bar{\theta})$ transform as follows (cf equations (3.3), (3.5) and (3.6))

$$
\begin{array}{ll}
\delta v_{2 j+1}(t)=-\mathrm{i} c_{2 j+1}(t) & \delta \varphi_{2 j+1}(t)=-\mathrm{i} \lambda_{2 j+1}(t) \\
\delta \bar{\psi}_{2 j+1}(t)=0 & \delta w_{2 j+1}(t)=\frac{1}{2} \dot{c}_{2 j+1}(t) \\
\delta v_{2 j+1}^{*}(t)=\mathrm{i} c_{2 j+1}^{*}(t) & \delta \bar{\varphi}_{2 j+1}(t)=\mathrm{i} \bar{\lambda}_{2 j+1}(t) \\
\delta \psi_{2 j+1}(t)=0 & \delta w_{2 j+1}^{*}(t)=\frac{1}{2} \dot{c}_{2 j+1}^{*}(t) .
\end{array}
$$

Observe that the $w$-term is supersymmetric and gauge invariant, transforming as a total derivative in both cases. The kinetic term is replaced by

$$
\sum_{j=1}^{N} X_{2 j+1} \exp \left(V_{2 j+1}+V_{2 i+1}^{*}\right) X_{2 j+1}^{*}+\sum_{j=1}^{N} X_{2 j} \exp \left(-V_{2 j-1}-V_{2 j+1}-V_{2 j-1}^{*}-V_{2 j+1}^{*}\right) X_{2 j}^{*}
$$

(3.8) is obviously invariant. From (3.7) it may be seen that we can go to a special gauge (in which supersymmetry is no longer manifest), such that

$$
v_{2 j+1}(t)=v_{2 j+1}^{*}(t)=0 \quad \text { and } \quad \varphi_{2 j+1}(t)=\tilde{\varphi}_{2 j+1}(t)=0
$$

and all the physics is contained in $\psi_{2 j+1}, \bar{\psi}_{2 j+1}$ and (the real part of) $w_{2 j+1}$. In terms of component fields

$$
\begin{aligned}
& \frac{\partial^{2}}{\partial \bar{\theta} \partial \theta} X_{2 j+1} \exp \left(+V_{2 j+1}+V_{2 j+1}^{*}\right) X_{2 j+1}^{*} \\
&= \frac{\partial^{2}}{\partial \bar{\theta} \partial \theta}\left\{( \overline { \chi } _ { 2 j + 1 } + \theta a _ { 2 j + 1 } ^ { * } + \frac { 1 } { 2 } \mathrm { i } \theta \overline { \theta } \dot { \overline { \chi } } _ { 2 j + 1 } ) \left[1+\theta \psi_{2 j+1}+\bar{\psi}_{2 j+1} \bar{\theta}\right.\right. \\
&\left.\left.+\theta \bar{\theta}\left(w_{2 j+1}+w_{2 j+1}^{*}-\bar{\psi}_{2 j+1} \psi_{2 j+1}\right)\right]\left(\chi_{2 j+1}+\bar{\theta} a_{2 j+1}-\frac{1}{2} \mathrm{i} \theta \bar{\theta} \dot{\chi}_{2 j+1}\right)\right\} \\
& \cong \dot{\mathrm{i}} \dot{\bar{\chi}}_{2 j+1} \chi_{2 j+1}+a_{2 j+1}^{*} a_{2 j+1}-a_{2 j+1}^{*} \bar{\psi}_{2 j+1} \chi_{2 j+1}-a_{2 j+1} \bar{\chi}_{2 j+1} \psi_{2 j+1} \\
&+\bar{\chi}_{2 j+1} \chi_{2 j+1}\left(w_{2 j+1}+w_{2 j+1}^{*}-\bar{\psi}_{2 j+1} \psi_{2 j+1}\right)
\end{aligned}
$$

$$
\begin{aligned}
\frac{\partial^{2}}{\partial \bar{\theta} \partial \theta} X_{2 j} \exp ( & \left.-V_{2 j-1}-V_{2 j+1}-V_{2 j-1}^{*}-V_{2 i+1}^{*}\right) X_{2 j}^{*} \\
\cong & \mathrm{i} \dot{\chi}_{2 j} \bar{\chi}_{2 j}+a_{2 j}^{*} a_{2 j}+a_{2 j}\left(\bar{\psi}_{2 j-1}+\bar{\psi}_{2 j+1}\right) \bar{\chi}_{2 j}+a_{2 j}^{*} \chi_{2 j}\left(\psi_{2 j-1}+\psi_{2 j+1}\right) \\
& \quad \bar{\chi}_{2 j} \chi_{2 j}\left[w_{2 j-1}+w_{2 j-1}^{*}+w_{2 j+1}+w_{2 j+1}^{*}+\left(\bar{\psi}_{2 j-1}+\bar{\psi}_{2 j+1}\right)\left(\psi_{2 j-1}+\psi_{2 j+1}\right)\right] .
\end{aligned}
$$

To construct an invariant containing gauge fields only we note that because of $\overline{\mathrm{D}} \Lambda_{2 i+1}=\mathrm{D} \Lambda_{2 j+1}^{*}=0$ and $(3.6) \dagger$

$$
\begin{aligned}
& \overline{\mathrm{D}} V_{2 j+1}(t, \theta, \bar{\theta})=\bar{\psi}_{2 j+1}(t)+\theta\left(w_{2 j+1}(t)-\frac{1}{2} \mathrm{i} \dot{v}_{2 j+1}(t)\right)+\frac{1}{2} \mathrm{i} \theta \bar{\theta} \dot{\bar{\psi}}_{2 j+1}(t) \\
& \mathrm{D} V_{2 j+1}^{*}(t, \theta, \bar{\theta})=\psi_{2 j+1}(t)+\bar{\theta}\left(w_{2 j+1}^{*}(t)+\frac{1}{2} \mathrm{i} \dot{v}_{2 j+1}^{*}(t)\right)-\frac{1}{2} \mathrm{i} \theta \bar{\theta} \dot{\psi}_{2 j+1}(t)
\end{aligned}
$$

are gauge independent. In the special gauge (3.9) $v, v^{*}$ drop out; a suitable kinetic term is given by (in special gauge)

$$
\frac{\partial^{2}}{\partial \bar{\theta} \partial \theta} \overline{\mathrm{D}} V_{2 j+1} \mathrm{D} V_{2 j+1}^{*} \cong \mathrm{i} \dot{\bar{\psi}} \dot{\psi}_{2 j+1} \psi_{2 j+1}+w_{2 j+1}^{*} w_{2 j+1}
$$

$\dagger$ In relativistic supersymmetry it was possible to choose $V=V^{*}$. Here, this turns out to be impossible, for to obtain a gauge field invariant we would have to apply $D, \bar{D}$ twice. However, $D^{2}=\bar{D}^{2}=0$. 
Thus, $w$ is an auxiliary variable. Adding (3.1), (3.10) and (3.12) we get the gauge invariant extension of (3.1) and use the equations of motion to eliminate $a$ and $w$ :

$$
\begin{aligned}
& -a_{2 j+1}^{*}=-\bar{\chi}_{2 j+1} \psi_{2 j+1}-\tilde{a}_{2 j+1}^{*}(\chi, \bar{\chi}) \\
& -a_{2 j}^{*}=\left(\bar{\psi}_{2 j-1}+\bar{\psi}_{2 j+1}\right) \bar{\chi}_{2 j}-\tilde{a}_{2 j}^{*}(\chi, \bar{\chi}) \\
& -w_{2 j+1}^{*}=\bar{\chi}_{2 j} \chi_{2 j}+\bar{\chi}_{2 j+1} \chi_{2 j+1}+\bar{\chi}_{2 j+2} \chi_{2 j+2}
\end{aligned}
$$

$\left(\tilde{a}_{2 j}^{*} \equiv a_{2 j}^{*}, \tilde{a}_{2 j+1}^{*} \equiv a_{2 j+1}^{*}\right.$ of equation (3.2)). Substituting we get

$$
\begin{aligned}
H=\sum_{j=1}^{2 N} \tilde{a}_{j}^{*}(\chi, \bar{\chi}) \tilde{a}_{j}(\chi, \bar{\chi})+\sum_{j=1}^{N}\left(\bar{\chi}_{2 j+1} \psi_{2 j+1} \tilde{a}_{2 j+1}(\chi, \bar{\chi})+\mathrm{HC}\right) \\
\quad-\sum_{j=1}^{N}\left\{\left(\bar{\psi}_{2 j-1}+\bar{\psi}_{2 j+1}\right) \bar{\chi}_{2 j} \tilde{a}_{2 j}(\chi, \bar{\chi})\right\}+\sum_{j=1}^{N} w_{2 j+1}^{*}(\chi, \bar{\chi}) w_{2 j+1}(\chi, \bar{\chi}) .
\end{aligned}
$$

(The first term just reproduces the Hamiltonian corresponding to the Lagrangian (3.1); note that $\tilde{a}=\tilde{a}^{*}=0$ if $g=0$ ). Ising-like interactions are contained in $w^{*} w$-this remains true in more than one dimension because, as was discussed in the introduction, undesired factors cancel; letting $g$ approach 0 a pure Ising model is obtained and gauge invariance generates a whole class of equivalent (and, in general, non-Isingtype) models.

To gauge the model of $\S 2$ we define $\left(j=\left(j_{1}, j_{2}\right)\right)$

$$
\begin{aligned}
& X_{2 j} \rightarrow \mathrm{e}^{\mathrm{i} \Lambda_{2 i}} X_{2 j} ; \quad X_{2 j+\hat{l}} \rightarrow \exp \left[-\mathrm{i}\left(\Lambda_{2 j}+\Lambda_{2 j+2 . \hat{1}}\right)\right] X_{2 j+\hat{1}} \\
& X_{2 j+\hat{\imath}} \rightarrow \exp \left[-\mathrm{i}\left(\Lambda_{2 j}+\Lambda_{2 j+2.2}\right)\right] X_{2 j+2}
\end{aligned}
$$

and similar calculations may be performed. Of course, the model we then obtain is more complicated than (3.14).

\section{Conclusion}

It remains to be seen what advantages and insights can be gained from supersymmetry and gauge symmetry as we have defined them. For the special examples discussed in the preceding sections there remains the intriguing possibility that one may be able to explicitly solve (i.e. diagonalise) a multi-spin interaction by adroitly exploiting its invariance under supersymmetry transformations (and, perhaps, gauge invariance). Beyond the present framework, a study of different and more complicated graded Lie algebras may also be useful, the algebra consisting of $Q, Q^{\dagger}$ and $H$ being the simplest example one can think of (Nahm et al 1977); in contrast to relativistic physics where all possibly relevant algebras have been classified (Haag et al 1975), there is as yet no such classification scheme in non-relativistic physics. The hope is, of course, that more complicated algebras might eventually lead to the construction of more realistic supersymmetric models, describing, for instance, an electron-phonon or any electronboson system, at least in a part of its spectrum. Even if these questions remain a matter of speculation for the time being, one should be aware of the increasingly important role that graded Lie algebras and anticommuting ' $c$-numbers' have come to play in physics (Berezin and Marinov 1975, 1976, Barducci et al 1976). 


\section{Acknowledgments}

It is a pleasure to thank Professor Wess for advice and encouragement. Helpful comments by one of the referees are also gratefully acknowledged.

\section{Appendix 1}

In this appendix the physical consequences and insights that may be extracted from supersymmetry will be discussed. We write $B$ for bosonic and $F$ for fermionic operators; $Q, Q^{+}$-the generators of supersymmetry-are of fermionic type. The 'parity' $(-1)^{p(a)}$ of a state $|a\rangle$ is then defined by

$$
p(a):=\left\{\begin{aligned}
+1 & \text { if }|a\rangle \text { fermionic } \\
0 & \text { if }|a\rangle \text { bosonic. }
\end{aligned}\right.
$$

In order to have $\{\zeta, F\}=[\zeta, B]=0$ where $\zeta$ is a Grassmann number, we have to stipulate that

$$
\zeta|a\rangle=(-1)^{p(a)}|a\rangle \zeta .
$$

Furthermore two types of trace operators must be introduced:

$$
\begin{array}{ll}
\operatorname{Tr} B:=\sum_{a}\langle a|B| a\rangle & \text { 'Bose trace' } \\
\widetilde{\operatorname{Tr}} B:=\sum_{a}(-1)^{p(a)}\langle a|B| a\rangle & \text { 'Fermi trace' }
\end{array}
$$

(note that $\langle a|F| a\rangle=0$ always). From (A.2) and (A.3) it follows easily that

$$
\operatorname{Tr} \zeta B=\zeta \widetilde{\operatorname{Tr}} B \quad \text { and } \quad \widetilde{\operatorname{Tr}} \zeta B=\zeta \operatorname{Tr} B .
$$

The cyclicity properties are

$$
\begin{aligned}
& \operatorname{Tr}\left[B_{1}, B_{2}\right]=\operatorname{Tr}\left[F_{1}, F_{2}\right]=0 \\
& \operatorname{Tr}\left[B_{1}, B_{2}\right]=\widetilde{\operatorname{Tr}}\left\{F_{1}, F_{2}\right\}=0 .
\end{aligned}
$$

In accordance with (A.3) two types of expectation functionals are defined:

$$
\langle B\rangle:=\frac{\operatorname{Tr} B \mathrm{e}^{-\beta H}}{\operatorname{Tr} \mathrm{e}^{-\beta H}} \quad \text { and } \quad\langle B\rangle^{\prime}:=\frac{\widetilde{\operatorname{Tr}} B \mathrm{e}^{-\beta H}}{\operatorname{Tr} \mathrm{e}^{-\beta H}}
$$

and again

$$
\langle\zeta B\rangle=\zeta\langle B\rangle^{\prime} \quad \text { and } \quad\langle\zeta B\rangle^{\prime}=\zeta\langle B\rangle .
$$

All identities among correlation functions for supersymmetry follow from

$$
\begin{aligned}
& \frac{\partial}{\partial \zeta}\langle[\zeta Q, F]\rangle=\langle\{Q, F\}\rangle^{\prime}=0 \\
& \frac{\partial}{\partial \zeta}\langle\{\zeta Q, F\}\rangle^{\prime}=\langle[Q, F]\rangle=0
\end{aligned}
$$

where $F$ is any Fermi-type operator that can be built from the fundamental operators 
$\left(\psi, \bar{\psi}, \chi, \bar{\chi}\right.$ in our examples). It is not known to the author whether the $\langle. .\rangle^{\prime}$ bracket has any significance in itself for

$$
\langle 1\rangle^{\prime}=0
$$

as follows from the basic algebra (cf I). In any case, we regard it as an auxiliary device to extract information from supersymmetry.

\section{Appendix 2}

To reconstruct operators $Q$ and $Q^{\dagger}$ from a given Lagrangian we note that

$$
\delta \mathscr{L}=\sum_{i}\left(\delta \varphi_{i} \frac{\partial \mathscr{L}}{\partial \varphi_{i}}+\delta \dot{\varphi}_{i} \frac{\partial \mathscr{L}}{\partial \dot{\varphi}_{i}}\right)=-\frac{\mathrm{d}}{\mathrm{d} t} K
$$

where $K$ is some function of $\varphi_{i}$, the fundamental variables appearing in $\mathscr{L}$ and of $\zeta, \bar{\zeta}$. If $\varphi$ anticommutes one has to be careful about the ordering of $\delta \varphi$ and $\partial / \partial \varphi$ as

$$
\delta \varphi \frac{\partial \mathscr{L}}{\partial \varphi}=-\frac{\partial \mathscr{L}}{\partial \varphi} \delta \varphi
$$

If $\partial / \partial \varphi$ is applied from left to right then $\delta \varphi(\partial / \partial \varphi)$ gives the correct result (otherwise $(\overleftarrow{\partial / \partial \varphi}) \delta \varphi$ ). Using equations of motion we obtain from (A.10)

$$
\sum_{i} \frac{\mathrm{d}}{\mathrm{d} t}\left(\delta \varphi_{i} \frac{\partial \mathscr{L}}{\partial \dot{\varphi}_{i}}\right)=-\frac{\mathrm{d}}{\mathrm{d} t} K
$$

Hence, the charges are

$$
\begin{aligned}
& Q=\frac{\partial}{\partial \zeta}\left(K+\sum_{i} \delta \varphi_{i} \frac{\partial \mathscr{L}}{\partial \dot{\varphi}_{i}}\right) \\
& Q^{+}=\frac{\partial}{\partial \bar{\zeta}}\left(K+\sum_{i} \delta \varphi_{i} \frac{\partial \mathscr{L}}{\partial \dot{\varphi}_{i}}\right) .
\end{aligned}
$$

For the model (3.1) this procedure leads to equation (3.1) of I.

\section{References}

Barducci A, Casalbuoni R and Lusanna L 1976 University of Florence Preprint Berezin F A and Marinov M S 1975 JETP Lett. 21320

1976 Institute for Theoretical and Experimental Physics Moscow Report No. 43

Ferrara S and Zumino B 1974 Nucl. Phys. B 79413

Haag R, Lopuszanski J T and Sohnius M 1975 Nucl. Phys. B 88257

Lieb E, Schultz T and Mattis D 1961 Ann. Phys., NY 16407

Nahm W, Rittenberg V and Scheunert M 1977 Bonn University Preprint

Nicolai H 1976 J. Phys. A: Math. Gen. 91497

Salam A and Strathdee J 1974 Nucl. Phys. B 76477 